\title{
The Role of Transdermal Testosterone Patch Over Pregnancy Rates in Poor Ovarian Responders Undergoing ICSI Cycles
}

Hassan FI, Shehata M, El-Sheikh W.A., Mostafa M.M. Younis*

Department of Obstetrics and Gynecology, Faculty of Medicine - Al-Azhar University

*Corresponding author: Mostafa M.M. Younis, Mobile: (+20)0101872334, E-Mail: abomousa29@yahoo.com

\begin{abstract}
Background: poor responder management in IVF represents a critical dispute for fertility specialists. Meanwhile, there is a lack of evidence to support the use of any particular intervention to improve treatment outcomes in poor responders because of the small sample size and the heterogeneity between the trials on the definition of poor ovarian responders (POR). Objective: the aim of this study was to evaluate the role of transdermal testosterone on the probability of pregnancy achievement in poor responders undergoing ICSI trials. Thus, the number of oocytes, pregnancy rates (clinical and live birth) and HMG dose and duration were compared between both trials.

Patients and Methods: This single-centre, randomized controlled trial was conducted from August 2016 to August 2017. A total of 61 poor responder patients with previous failed trial underwent their second ICSI trial. The patients received ovarian stimulation using a short GnRH agonist protocol in both trials with transdermal testosterone patches being used in the second trial starting five days prior to HMG.

Results: The oocytes recruitment and the pregnancy rates were higher after testosterone pretreatment in the second trial, however, the difference between the previous and the second trial didn't reach the statistical significance.

Conclusion: Testosterone patches effect on oocyte number and pregnancy rates appears to be minimal for poor responders. However, further trials are needed to prove the study's conclusion in the future.

Keywords: Transdermal Testosterone Patch, Over Pregnancy Rates, Ovarian Responders Undergoing ICSI Cycles.
\end{abstract}

\section{INTRODUCTION}

A woman is born with about 2 million primordial follicles, yet by the onset of menarche only about 400,000 follicles are left due to natural follicular atresia ${ }^{(\mathbf{1})}$. As a woman reaches her mid-30s the pace of oocyte depletion begins to increase and by the time she reaches her late $30 \mathrm{~s}$, the number of follicles declines to approximately 25,000 , concomitant with a significant increase in miscarriage rate. The term "ovarian reserve" has traditionally been used to describe a woman's reproductive potential specifically, the number and quality of oocytes she possesses. However, commonly used ovarian reserve markers serve as a proxy for oocyte quantity but are considered poor predictors of oocyte quality ${ }^{(\mathbf{1})}$.

The treatment modalities for poor responders have shown a diversity across the literature. The need for an adjuvant therapy to solve the obstacle of poor ovarian response for maximal stimulation has gained much interest recently; thus, the trials over growth hormone, androgens and aspirin have gained much popularity. Androgen appears to be positive regulators of follicular development and also show some synergistic effects with FSH on folliculogenesis. Remarkably, it has been reported that treatment of rhesus monkeys with dihydrotestosterone or testosterone augments follicular FSH receptor expression in granulosa cells ${ }^{(2)}$, promotes initiation of primordial follicle growth and increases the number of growing pre-antral and small antral follicles ${ }^{(3)}$

\section{Aim of the work:}

The primary aim of this study is to evaluate the efficacy of testosterone when administered via transdermal route in improving the pregnancy rates in poor responder patients undergoing ICSI trials versus the traditional controlled ovarian stimulation protocols without any adjuvant therapy given. The secondary aim is to evaluate the safety profile (common sideeffects) of this drug when used prior to ICSI trials.

\section{PATIENTS AND METHODS}

Sixty one poor responders fulfilling the Bologna criteria were enrolled in this prospective, clinical trial. The study was conducted at The International Islamic Center for Population Studies and Research (IICPSR), Al-Azhar University from August 2016 to August 2017.

Patients with previous failed trial due to poor ovarian response were allocated and prescribed the transdermal testosterone patches and results were compared with the first trial for the same patients. Patient's main criteria were menstruating females aged 31-39 years at the time of their second IVF attempt with a background of failed or cancelled IVF cycle at our fertility unit because of poor follicular response. The main indications were: unexplained infertility, tubal infertility and minimal to mild endometriosis. Exclusion criteria included females over 39 years, male factor infertility, endocrinal disorders and inverted $\mathrm{FSH} / \mathrm{LH}$ ratio.

The study was approved by the Ethics Committee of the international Islamic Center for Population Studies and Research, Al-Azhar University. Written consents were obtained from all patients.

Ovarian stimulation:

All patients underwent a short stimulation protocol with $\mathrm{GnRH}$ agonist triptorelin $0.1 \mathrm{mg}$ starting 
on the first day of the menstrual cycle, followed daily HMG injections ranging from 375 IU to $450 \mathrm{IU}$ starting from the second day of menstrual cycle. The testosterone transdermal patch $(2.5 \mathrm{mg})$ was applied to the inner side of the thigh 5 days prior to stimulation protocols. Patients in the previous trial underwent the same protocol without receiving testosterone pretreatment. Triggering of final oocyte maturation was performed using $1000 \mathrm{IU}$ IM of hCG as soon as at least three follicles reached $17 \mathrm{~mm}$ in diameter or if this was not possible, when the maximum number of follicles were present

\section{IVF procedure and luteal phase support:}

Oocyte retrieval was performed by ultrasoundguided transvaginal follicular aspiration $36 \mathrm{~h}$ after hCG administration. Intracytoplasmic sperm injection (ICSI) was performed in all patients. Luteal phase was supported by administration of $400 \mathrm{mg} /$ day (vaginal suppositories) starting on the day of oocyte retrieval and continued up to the day of pregnancy test or in case of a positive test until the seventh week of pregnancy.

\section{Hormonal measurements and ultrasound assessment of follicular development:}

Hormonal profile determination was done to all cases at the second or third day of their menstrual cycles.

\section{Outcome measures:}

The primary outcome measure was the number of oocytes retrieved, duration and dose of stimulation, clinical pregnancy rate, live birth rate and cancellation rate between both trials

Secondary outcome measures included any possible local or systemic side effects of testosterone patches in the form of skin reaction at the site of application, increased hair growth, nausea, acne or voice deepening.

\section{Primary outcome measure:}

Using analysis per intention to treat (ITT), there was no statistically significant difference was found between the current trial and the previous trial regarding the number of retrieved oocytes, dose and duration of HMG, clinical and live birth rate

\section{Secondary outcome measure:}

No side effects, such as skin reaction at the site of application, increased hair growth, nausea, acne or voice deepening, were reported in testosterone treated patients for the duration of follow-up.

\section{Statistical analysis:}

The results were recorded, tabulated and statistically analyzed. The data of all patients were fed into an IBM- compatible personal computer and analyzed using SPSS (version 15.0., 2006). For qualitative characteristics, the number and percentage of women with these characteristics were calculated, for quantitative characteristics, range, mean and standard deviations were calculated. Comparative statistics were done among different groups using analysis of variance. $\mathrm{P}$ value $<0.05$ was considered significant, the $95 \%$ confidence intervals (95\% C.I.) of various groups were determined.

\section{RESULTS}

No statistically significant differences were observed after testosterone pretreatment in the current trial and the previous trial without testosterone regarding baseline patient characteristics.

Table (1): Baseline characteristics of patients in the previous trial:

\begin{tabular}{|l||l|}
\hline \multicolumn{1}{|c||}{ VARIABLE } & VALUE \\
\hline \hline \multicolumn{1}{|c|}{ Demographic characteristic } \\
\hline \hline Age (mean \pm SD): & $32.31 \pm \mathbf{4 . 1}$ \\
\hline \hline Infertility type n $(\%)$ & \\
Primary & $\mathbf{4 6}(\mathbf{7 5 . 4} \%)$ \\
Secondary & $\mathbf{1 5}(\mathbf{2 4 . 6} \%)$ \\
Total & $\mathbf{6 1}(\mathbf{1 0 0 \% )}$ \\
\hline \hline Infertility duration (mean \pm SD): & $\mathbf{5 . 8 5} \pm \mathbf{2 . 4}$ \\
\hline \hline BMI (mean \pm SD): & $\mathbf{2 9 . 1 6 \pm 2 . 2}$ \\
\hline \hline Baseline endocrine profile and antral follicle count \\
\hline \hline AFC (mean \pm SD): & $\mathbf{3 . 9 2} \pm \mathbf{0 . 7}$ \\
\hline \hline AMH (mean \pm SD): & $\mathbf{0 . 4 2} \pm \mathbf{0 . 1}$ \\
\hline \hline FSH (mean \pm SD): & $\mathbf{9 . 4 5} \pm \mathbf{1 . 5}$ \\
\hline
\end{tabular}

Table (1) shows that mean age of females in the first ICSI trial was $(32.31 \pm 4.1)$.Most of the studied females in the first trial had primary infertility. Mean infertility duration was $(5.85 \pm 2.4)$. Mean of AFC, AMH was (3.92 $\pm 0.7),(0.42 \pm 0.1)$ respectively.

Table (2): Baseline characteristics of patients in the second trial:

\begin{tabular}{|c|c|}
\hline Value & Variable \\
\hline \multicolumn{2}{|c|}{ Demographic characteristic } \\
\hline $33.72 \pm 3.0$ & Age $($ mean $\pm \mathrm{SD})$ \\
\hline & Infertility type $\mathrm{n}(\%)$ \\
\hline $45(73.8 \%)$ & Primary \\
\hline $16(26.2 \%)$ & Secondary \\
\hline $61(100 \%)$ & Total \\
\hline $7.38 \pm 2.2$ & $\begin{array}{l}\text { Infertility duration (mean } \pm \\
\text { SD) }\end{array}$ \\
\hline $29.21 \pm 2.3$ & $\mathrm{BMI}($ mean $\pm \mathrm{SD})$ \\
\hline \multicolumn{2}{|c|}{ Baseline endocrine profile and antral follicle coun } \\
\hline $3.79 \pm 0.7$ & $\mathrm{AFC}($ mean $\pm \mathrm{SD})$ \\
\hline $0.38 \pm 0.1$ & $\mathrm{AMH}($ mean $\pm \mathrm{SD})$ \\
\hline $10.04 \pm 1.4$ & FSH $($ mean \pm SD) \\
\hline
\end{tabular}

Table (2) Shows that mean age of females in the second ICSI trial was $(33.72 \pm 3)$. Most of the studied females in the second trial had infertility primary type. Mean infertility duration was $(7.38 \pm$ 2.2). Mean of AFC, AMH was (3.79 \pm 0.7$),(0.38 \pm 0.1)$ respectively. 
Table (3): HMG dose and duration in the previous trial and after testosterone treatment in the second trial:

\begin{tabular}{|c|c|c|c|c|}
\hline \multirow{2}{*}{ Variable } & \multirow{2}{*}{$\begin{array}{c}\text { First trial group } \\
\qquad \begin{array}{c}(\mathrm{N}=61) \\
(\mathrm{Mean} \pm \mathrm{SD})\end{array}\end{array}$} & \multirow{2}{*}{$\begin{array}{c}\text { Second trial } \\
\text { (testosterone) group } \\
(\mathrm{N}=61) \\
(\text { Mean } \pm \text { SD })\end{array}$} & \multicolumn{2}{|c|}{ Significance } \\
\hline & & & $\mathbf{T}$ & $\mathbf{p}$ \\
\hline HMG dose & $436.48 \pm 29.1$ & $437.7 \pm 27.9$ & 0.24 & 0.8 \\
\hline HMG duration & $12.25 \pm 1.3$ & $12.08 \pm 1.1$ & 0.76 & 0.45 \\
\hline
\end{tabular}

Table (3) shows that mean of HMG dose in pretreatment group was (436.48 \pm 29.1$)$ vs. (437.7 \pm 27.9$)$ (p value 0.8 )in the control group, there was no statistically significant difference between both groups .Mean of HMG duration was about (12 days) in both groups ( $\mathrm{p}$ value 0.45 ).

Table (4): Outcome of ovarian stimulation in the first and second trials:

\begin{tabular}{|c|c|c|c|c|}
\hline \multirow{2}{*}{ VARIABLE } & \multirow{2}{*}{$\begin{array}{c}\text { PREVIOUS } \\
\text { TRIAL } \\
(\mathbf{N}=61) \\
(\mathrm{MEAN} \pm \mathrm{SD})\end{array}$} & \multirow{2}{*}{$\begin{array}{c}\text { SECOND TRIAL } \\
(\mathbf{N}=\mathbf{6 1}) \\
(\mathrm{MEAN} \pm \mathrm{SD})\end{array}$} & \multicolumn{2}{|c|}{ SIGNIFICANCE } \\
\hline & & & $\begin{array}{c}\mathrm{t} \\
\text { for paired } \\
\text { difference }\end{array}$ & $\mathbf{P}$ \\
\hline No. of follicles & $4.13 \pm 1.1$ & $4.15 \pm 0.9$ & 0.11 & 0.91 \\
\hline No of oocytes & $3.1 \pm 1.01$ & $3.21 \pm 0.9$ & $\begin{array}{ll}\mathbf{0 . 0 1} \\
\end{array}$ & 0.99 \\
\hline $\mathrm{M} 2$ & $0.89 \pm 0.7$ & $0.93 \pm 0.7$ & 0.5 & 0.62 \\
\hline M1 & $1.28 \pm 0.6$ & $1.1 \pm 0.6$ & 1.9 & 0.06 \\
\hline $\mathrm{GV}$ & $0.8 \pm 0.6$ & $0.85 \pm 0.7$ & 0.44 & 0.67 \\
\hline Atretic & $0.28 \pm 0.6$ & $0.32 \pm 0.4$ & 0.13 & 0.82 \\
\hline
\end{tabular}

Table (4) shows that there was a higher number of oocytes recruited in the second trial. However, no statistically significant difference was found between first and second trial regarding outcome of ovarian stimulation.

Table (5): Pregnancy outcome in the first and second trial:

\begin{tabular}{|c|c|c|c|c|}
\hline \multirow[t]{2}{*}{ VARIABLE } & \multirow{2}{*}{$\begin{array}{l}\text { FIRST TRIAL } \\
(\mathrm{N}=61)\end{array}$} & \multirow{2}{*}{$\begin{array}{l}\text { SECOND TRIAL } \\
(\mathrm{N}=61)\end{array}$} & \multicolumn{2}{|c|}{ SIGNIFICANCE } \\
\hline & & & $\overline{\mathbf{Z}}$ & $\overline{\mathbf{P}}$ \\
\hline Patients with embryo transfer & $54(88.5 \%)$ & $55(90.2 \%)$ & -0.29 & 0.77 \\
\hline "Cancelation rate & $7(11.5 \%)$ & $6(9.8 \%)$ & 0.29 & 0.77 \\
\hline Clinical pregnancy & $3(4.9 \%)$ & $5(8.2 \%)$ & \begin{tabular}{c|}
$0.73-$ \\
\end{tabular} & 0.47 \\
\hline $\begin{array}{l}\text { Clinical pregnancy per embryo } \\
\text { transfer }\end{array}$ & $3(5.6 \%)$ & $5(9.1 \%)$ & 0.7- & 0.48 \\
\hline Live birth rate & 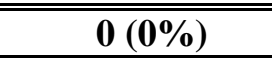 & $3(4.9 \%)$ & \begin{tabular}{c|}
$1.75-$ \\
\end{tabular} & 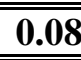 \\
\hline Live birth per embryo transfer & $\mathbf{0}(0 \%)$ & $3(5.5 \%)$ & 1.74- & $\mathbf{0 . 0 8}$ \\
\hline
\end{tabular}

Table (5) shows that proportion of clinical pregnancy occurrence was higher in second trial than the first trial, the difference between groups wasn't statistically significant. No significant difference was observed regarding live birth rate before the testosterone pretreatment in the first trial and the after pretreatment with testosterone in the second trial. 


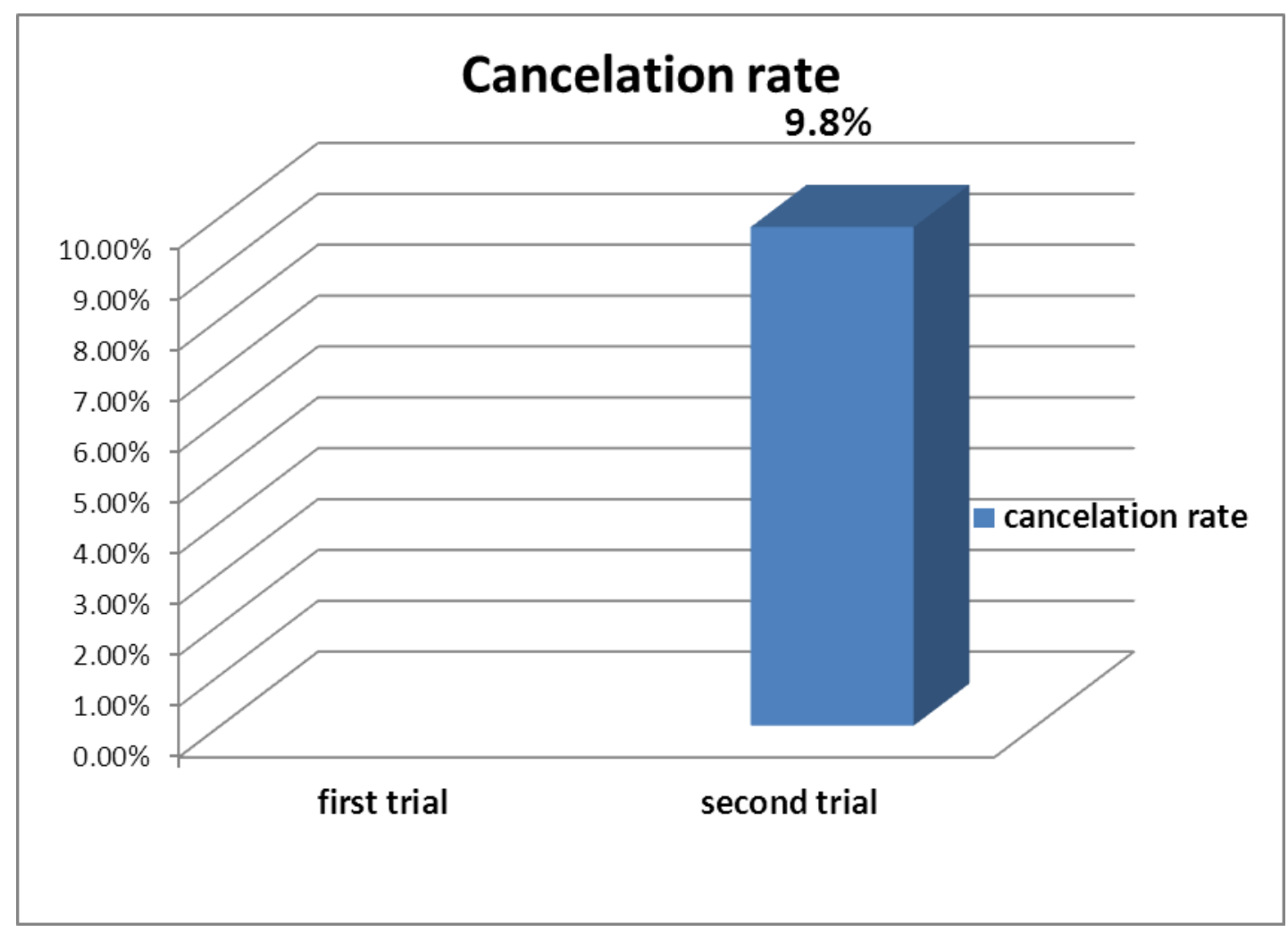

Figure (1): Cancelation rate in the first and second trials

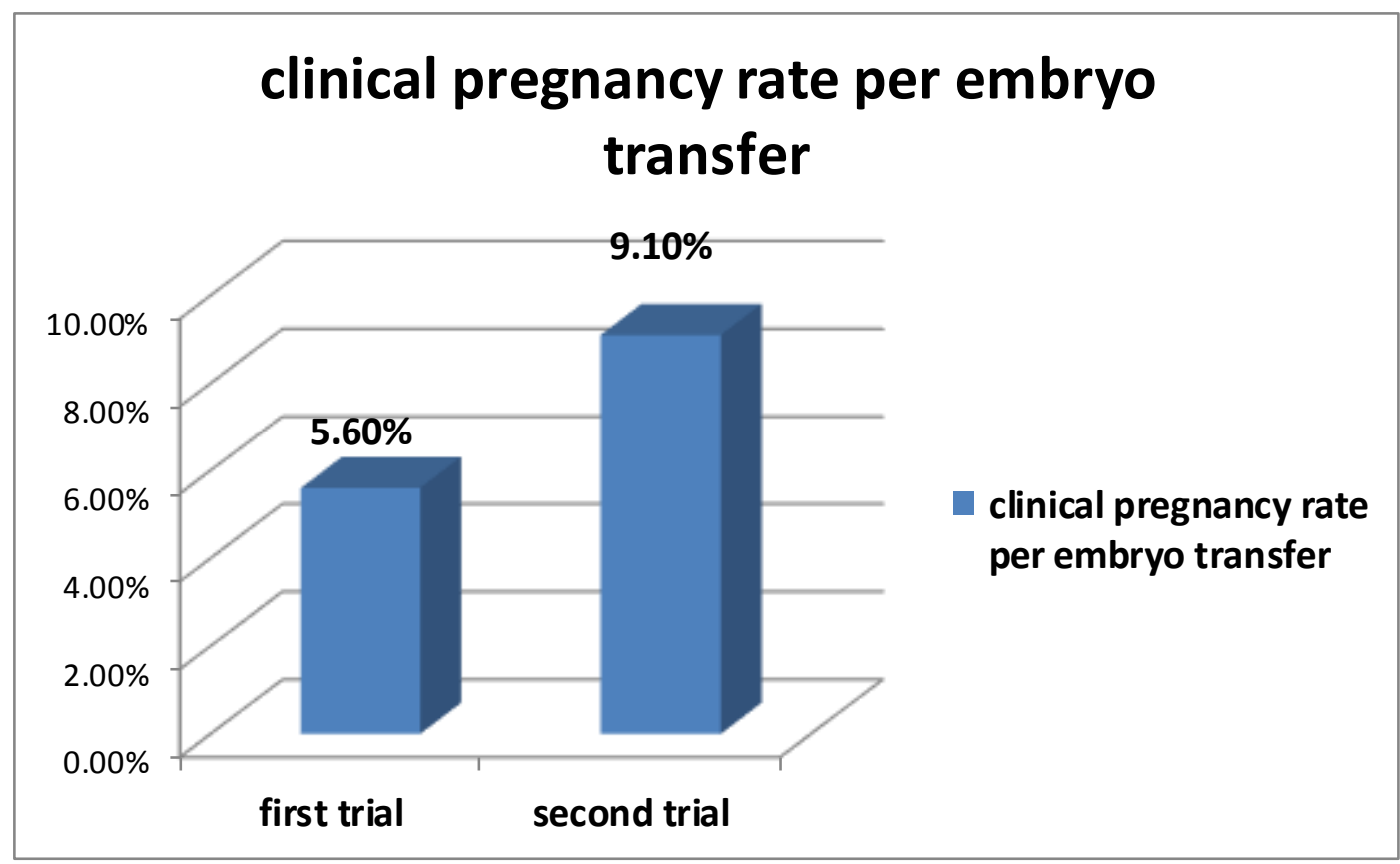

Figure (2): Clinical pregnancy rate per embryo transfer

\section{DISCUSSION}

The current study has shown that transdermal testosterone pretreatment at a dose of $2.5 \mathrm{mg} /$ day for 5 days prior to ovarian stimulation did not increase the number of oocytes retrieved, clinical pregnancy and live birth rate in poor responders undergoing ICSI. Despite the presence of a significantly higher testosterone level, as expected after 5 days of testosterone administration compared with the previous trial, no statistically significant increase in the retrieved number of oocytes, clinical pregnancy and live birth rate was observed. 
No such difference was also present in the number of antral follicles at initiation of stimulation between the two groups compared.

As regards the mean number of oocytes retrieved; there was a higher mean number of oocytes retrieved after testosterone pretreatment in comparison with the previous trial $(4.15 \pm 0.9$ versus $4.13 \pm 1.1, \mathrm{p}$ value 0.99 ) but with no significant difference between the two groups.

Bosdou et al. (4), in a study included 50 patients revealed that transdermal testosterone pretreatment at a dose of $10 \mathrm{mg} / \mathrm{day}$ for 21 days does not increase the number of oocytes retrieved by more than 1.5 in poor responders undergoing ICSI, stimulated by recombinant $\mathrm{FSH}$ in a long GnRH agonist protocol. This conclusion is in agreement with our study.

Massin et al. ${ }^{(5)}$, in a study that included 49 patients, showed a non-significant increase with testosterone pretreatment $(10 \mathrm{mg} /$ day for $15-21$ days) as compared with no pretreatment on the number of oocytes retrieved (mean difference: $+0.31 \mathrm{COCs}, 95 \%$ CI: -1.64 to +2.26 ). This study is on agreement with our work.

On the contrary of our study, Kim et al. ${ }^{(6)}$, in a large RCT including 110 patients showed that testosterone pretreatment $(12.5 \mathrm{mg} / \mathrm{day}$ for 21 days) significantly increased the number of oocytes retrieved (mean difference: $+1.60 \mathrm{COCs}, 95 \% \mathrm{CI}:+0.97$ to +2.23 ) in poor responders as compared with no pretreatment. The same author in 2014 Kim et al. ${ }^{(7)}$, studied the effect of testosterone pretreatment in 120 poor responders relatively to the duration of its administration (no pretreatment, 2, 3, and 4 weeks of testosterone pretreatment). This study suggested that testosterone has a beneficial effect on the number of COCs retrieved, which however, is statistically detectable as compared with no treatment only after 3 or 4 weeks of administration. The mean+SD of oocytes retrieved in the four groups studied were: no pretreatment: $3.9+1.3,2$ weeks pretreatment: $4.3+1.6$, 3 weeks pretreatment: $5.3+2.0$, 4 weeks pretreatment: 5.8+1.9.

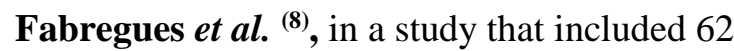
poor responder patients who were randomized in two treatment groups in their second IVF attempt. In patients in Group $1(\mathrm{n}=31)$, transdermal application of testosterone preceding standard gonadotrophin ovarian stimulation under pituitary suppression was used. In Group 2 ( $n=31$ patients), ovarian stimulation was carried out with high-dose gonadotrophin in association with a mini-dose GnRH agonist protocol. The number of patients with ovum retrieval tended to be higher in Group 1 than in Group 2 (80.6\% versus $58.1 \%, \mathrm{P}=0.09)$, the difference reaching statistical significance $(81.2 \%$ versus $41.1 \%, \mathrm{P}<0.05)$ when only patients having normal basal FSH levels (16 and
17 patients in Groups 1 and 2, respectively) were considered.

As regards the live birth rate per embryo transfer:

The percentage of positive live birth was higher after testosterone pretreatment (0/54 vs $3 / 55)$; however, the difference between both groups was statistically insignificant ( $\mathrm{p}$ value 0.08 ).

Massin et al. ${ }^{(5)}$, in agreement with our study, reported that only 2 out of 27 in pretreatment group versus 1 out of 26 patients of the control group had a live birth.

Bosdou et al. ${ }^{(4)}$ also in agreement with our work, revealed a live birth rate of $10 \%$ in pretreatment group (2 out of 26 ) and $9.5 \%$ in control group $(2$ out of 24) (PV 1.0).

Fábregues et al. ${ }^{\left({ }^{(8)}\right.}$ observed six live births in the intervention group $(\mathrm{n}=31)$ compared with four live births in the control group $(\mathrm{n}=31)$, whereas Kim et al. ${ }^{(6)}$ reported $15(\mathrm{n}=55)$ and seven $(\mathrm{n}=55)$, respectively.( Kim et al.2014), reported that The numbers live birth rates were significantly higher in 4 weeks TTG treatment group than those in control group in the previous 1,2 and 3 weeks $30.0 \%(9 / 30)$ versus $6.7 \%(2 / 30), 13.4 \%(4 / 30)$ and $20.0 \%(6 / 30)$ respectively.

As regards the clinical pregnancy rate per embryo:

The clinical pregnancy rate per embryo transfer after testosterone treatment was $9.1 \%$ (5 cases out of 55) higher than the clinical pregnancy rate in the previous trial which was $5.6 \%$ (3 cases out of 54); however, the difference did not reach the level of statistical significance ( $\mathrm{p}$ value 0.48 ).

Massin et al. ${ }^{(5)}$, revealed that The clinical pregnancy rate and implantation rate are reported to be one and four clinical pregnancies in placebo- and testosterone-treated patients, respectively, the low number of patients enrolled in each group precluded any statistical comparison ( $\mathrm{p}$ value 0.83 ).

Bosdou et al. ${ }^{(4)}$, reported that the clinical pregnancy rate was found to be $7.7 \%$ ( two cases out of 26) in the pretreatment group meanwhile the control group had a pregnancy rate of $8.3 \%$ (two cases out of 24) ( $p$ value 1) which is in agreement with our study.

Fábregues et al. (8) reported six clinical pregnancies in the intervention group $(n=31)$ compared with four clinical pregnancies in the control group $(n=31)(p$ value $=1)$ a possible explanation was presented on the basis of low FSH values in the patients of this study.

Kim et al. (6) found that The clinical pregnancy rates per cycle initiated and per cycle ET were also significantly higher in the TTG pretreatment group than in the control group $(\mathrm{P}=.041$ and $\mathrm{P}=.045$, respectively). Kim $\boldsymbol{e t}$ al. ${ }^{(7)}$ reported a rising clinical pregnancy rate with longer testosterone pretreatment ,so the clinical pregnancy rate was highest after 4 weeks therapy $36.7 \%$ (11/30) in comparison to $10.0 \%$ 
$(3 / 30), 16.7 \%(5 / 30), 30.0 \%(9 / 30)$ after the 1 st, 2 nd 3rd weeks respectively.

Regarding the HMG dose and duration:

In our work the mean of HMG dose after testosterone application in the second trial was (436.48 $\pm 29.1)$ vs. $(437.7 \pm 27.9)$ in the first trial, there was no statistically significant difference between both groups ( $p$ value $=0.8$ ). Mean of HMG duration was around 12 days in both groups as well ( $\mathrm{p}$ value $=0.45$ ).

Bosdou et al. ${ }^{(4)}$, in agreement with our work, reported that there was no statistical significant difference in the HMG dose (3750 versus 3600) (p value 0.65 ) or duration (12 versus 12.5 days) ( $\mathrm{p}$ value 0.65 ) between the pretreatment and the control group.

Massin et al. (5) reported no statistical significant difference between the control group and the intervention group regarding the HMG dose $(3955 \pm 234$ versus $3539 \pm 320$ unit)( $p$ value 0.11$)$ which is in agreement with our study, and the duration of HMG was also comparable ( $p$ value 0.87 ).

Fábregues et al. ${ }^{(8)}$ revealed that the total dose of FSH used was $2924 \pm 987$ in the pretreatment group in comparison to $3785 \pm 1321$ in the control group (p value <0.05). In addition, the duration of the gonadotrophin therapy needed to achieve the criteria for hCG injection were significantly lower in Group 1 this could be explained by the beneficial effect of testosterone application on the ovarian response among patients having normal basal FSH levels.

Kim et al. ${ }^{(6)}$ reported that the total dose of rhFSH required for oocytes were significantly lower in the pretreatment group than in the control group $(2,552.3 \pm 397$ versus $3,000.8 \pm 449)$ both $(\mathrm{P}<.001)$, and the duration of $\mathrm{GnRH}$ antagonist administration was also shorter in the pretreatment group $(\mathrm{P}=.001)$.

Kim et al. ${ }^{(7)}$ stated that the doses of HMG were significantly lower in the intervention groups after 4weeks,3weeks and 2 weeks pretreatment in comparison to the control group $(2,765.7 \pm 567.8$, $2,643.5 \pm 389,2,596.7 \pm 335.3$ versus $3,025.0 \pm 425.9)(\mathrm{p}$ value $<0,001)$ and the duration of HMG administration was also shorter in the pretreatment group $(\mathrm{P}<.001)$.

\section{Regarding the adverse effects}

There was no relevant side effects could be detected such as skin reaction at the site of application, increased hair growth, nausea, acne or voice deepening ,were reported after testosterone treatment for the duration of follow-up.

Kim et al. ${ }^{(7)}$ stated that after a 4-week period of testosterone pretreatment, there were no detectable side effects. However, it is not known whether such side effects would occur by using an increased testosterone dosage or a longer pretreatment period.

\section{CONCLUSION AND RECOMMENDATIONS}

After reviewing reports in the literature regarding the efficacy and safety of the use of transdermal testosterone patches prior to ICSI cycles in poor responders compared to the usual protocols, and after presenting and discussing the results of the current study, we can conclude the following:

The current study has shown that transdermal testosterone pretreatment at a dose of $2.5 \mathrm{mg} / \mathrm{day}$ for 5 days did not significantly increase the number of retrieved oocytes, clinical pregnancy rate or live birth rate in poor responders undergoing ICSI trials, stimulated by short GnRH agonist protocol.

Testosterone supplementation is considered a simple adjuvant therapy in poor responder patients, however, the efficacy of this therapy is still doubtful.. Therefore, further studies are needed to document the probable benefit from testosterone usage in clinical practice in poor responder patients undergoing ICSI trials.

\section{REFERENCES}

1. Practice Committee of the American Society for Reproductive Medicine (2015): Testing and interpreting measures of ovarian reserve: a committee opinion. Fertil Steril., 103: 9-17.

2. Vendola KA, Zhou J, Adesanya OO et al. (1998): Androgens stimulate early stages of follicular growth in the primate ovary. J Clin Invest., 101:2622-2629.

3. Weil S, Vendola K, Zhou J et al. (1999): Androgen and follicle-stimulating hormone interactions in primate ovarian follicle development. J Clin Endocrinol Metab., 84:2951-2956.

4. Bosdou JK, Venetis CA, Dafopoulos KZ et al. (2016): Transdermal testosterone pretreatment in poor responders undergoing ICSI: a randomized clinical trial. Hum Reprod., 31(5): 977-985.

5. Massin N, Cedrin-Durnerin I, Coussieu $\mathrm{C}$ et al. (2006): Effects of transdermal testosterone application on the ovarian response to FSH in poor responders undergoing assisted reproduction technique- $\mathrm{a}$ prospective, randomized, double-blind study. Hum Reprod., 21:1204-1211.

6. Kim CH, Howles CM, Lee HA (2011): The effect of transdermal testosterone gel pretreatment on controlled ovarian stimulation and IVF outcome in low responders. Fertil Steril., 95:679-683.

7. Kim CH, Ahn JW, Moon JW et al. (2014): Ovarian features after 2 weeks, 3 weeks and 4 weeks transdermal testosterone gel treatment and their associated effect on IVF outcomes in poor responders. Balsaenggwa Saengsig., 18:145-152

8. Fábregues F, Peñarrubia J, Creus $M$ et al. (2009): Transdermal testosterone may improve ovarian response to gonadotrophins in low-responder IVF patients: a randomized, clinical trial. Hum Reprod., 24: 